\title{
Fatores que afetam a ventilação com o reanimador manual autoinflável: uma revisão sistemática
}

\author{
Factors affecting manual resuscitator use: a systematic review
}

Pricila Mara N. de Oliveira ${ }^{1}$, Armando Augusto Almeida-Junior ${ }^{2}$, Celize Cruz B. Almeida ${ }^{3}$, Maria Ângela G. de O. Ribeiro ${ }^{4}$, José Dirceu Ribeiro ${ }^{5}$

\section{RESUMO}

Objetivo: O reanimador manual autoinflável é um dispositivo que fornece ventilação com pressão positiva. Pesquisas mostram que, apesar da padronização dos reanimadores manuais autoinfláveis pela American Society for Testing and Materials, diversos fatores afetam o desempenho da ventilação, porém, os resultados são conflitantes. O objetivo desse estudo foi verificar as evidências dos fatores que influenciam a ventilação pulmonar com reanimadores manuais infantil/ adulto por meio de uma revisão sistemática da literatura.

Fontes de dados: Foram incluídos artigos indexados nas bases Medline, Lilacs e SciELO publicados entre janeiro de 1986 e março de 2011. Utilizaram-se as palavras-chaves: "reanimador manual”, "ressuscitador manual", "ventilação manual", "ventilação com pressão positiva”, em inglês e português, além de "bag-valve".

Síntese dos dados: Foram selecionados 45 artigos, sendo a maioria experimental. Os trabalhos compararam os reanimadores manuais por marcas, modelos e analisaram as características dos profissionais que os utilizam. Estudos verificaram que a eficácia da ventilação com os reanimadores manuais depende da marca, modelo e características funcionais do aparelho utilizado, assim como formação, treinamento e experiência do profissional que os manipula. Outros fatores que podem influenciar são a forma de compressão dos rea-

Instituição: Departamento de Pediatria da Faculdade de Ciências Médicas da Universidade Estadual de Campinas (Unicamp), Campinas, SP, Brasil ${ }^{1}$ Mestre em Saúde da Criança e do Adolescente pela Unicamp; Fisioterapeuta do Serviço de Pediatria e Neonatologia do Hospital São Paulo da Universidade Federal de São Paulo (Unifesp), São Paulo, SP, Brasil

2Mestre em Saúde da Criança e do Adolescente pela Unicamp; Médico Intensivista Pediátrico do Hospital de Clínicas da Unicamp, Campinas, SP, Brasil

${ }^{3}$ Doutora em Saúde da Criança e do Adolescente pela Unicamp; Fisioterapeuta do Hospital de Clínicas da Unicamp, Campinas, SP, Brasil

${ }^{4}$ Doutora em Saúde da Criança e do Adolescente pela Faculdade de Ciências Médicas da Unicamp; Coordenadora dos Cursos de Pós-graduação em Fisioterapia Pediátrica da Faculdade de Ciências Médicas da Unicamp, Campinas, SP, Brasil

${ }^{5}$ Professor Associado Livre-Docente do Departamento de Pediatria da Faculdade de Ciências Médicas da Unicamp, Campinas, SP, Brasil nimadores manuais, o uso da válvula limitadora de pressão e o fluxo de oxigênio fornecido aos aparelhos.

Conclusões: A variabilidade nos parâmetros ventilatórios fornecidos durante a ventilação com reanimadores manuais não permite uniformizar a técnica, o que prejudica a reanimação cardiopulmonar. Apesar da maioria dos reanimadores manuais parecer estar de acordo com padrões internacionais, os equipamentos devem ser avaliados antes de utilizados no ambiente clínico. Pouco se sabe sobre os modelos pediátricos e neonatais.

Palavras-chave: ventilador; ressuscitação cardiopulmonar; ventilação pulmonar.

\section{ABSTRACT}

Objective: The manual resuscitator $(\mathrm{RM})$ is a device that provides positive pressure ventilation. Surveys conducted to assess the adequacy of manual resuscitators to American Society for Testing and Materials standards show that several factors affect manual ventilation. However, results are conflicting. The aim of this study was to verify evidence of factors that influence pediatric/adult pulmonary ventilation with manual resuscitator by a systematic review.

Data source: Original articles indexed in Medline, Lilacs and SciELO published from January 1986 to March 2011.

Endereço para correspondência:

Pricila Mara N. de Oliveira

Rua Tessalia Vieira de Camargo, 126

Caixa Postal 6111

CEP 13083-887 - Campinas/SP

E-mail: pricilaufjf@yahoo.com.br

Conflito de interesse: nada a declarar

Fonte financiadora: bolsa de mestrado da Coordenação de Aperfeiçoamento de Pessoal de Nível Superior (CAPES)

Recebido em: 4/11/2010

Aprovado em: 20/5/2011 
The key-words used were: "manual resuscitator", "manual ventilation", "positive pressure ventilation" in Portuguese and English, as well as "bag-valve".

Data Synthesis: 45 articles were selected, most of them experimental. The studies compared manual resuscitator brands and models, and analyzed the physical characteristics of professionals. The effectiveness of ventilation with manual resuscitator depends on the brand, model and functional characteristics of the equipment. Ventilation also varies with the education, training and experience of the professional who handles the equipment. Other factors that can influence effectiveness are the manual resuscitator compression form, the use of a pressure relief valve and the flow of oxygen provided to the manual resuscitator.

Conclusions: The variability of ventilatory parameters during manual resuscitation does not allow a standardization of the technique, being harmful to cardiopulmonary resuscitation. Although most manual resuscitator seem to follow international standards, the equipment must be evaluated in the clinical settings. There are few studies about pediatric and neonatal manual resuscitator models.

Key-words: ventilator; cardiopulmonary resuscitation; pulmonary ventilation.

\section{Introdução}

O reanimador manual autoinflável (RM) é também conhecido como bolsa autoinflável, ventilador manual, hiperinsuflador e dispositivo bolsa-válvula. É utilizado para fornecer ventilação com pressão positiva a pacientes com necessidade de suporte ventilatório ${ }^{(1)}$, sendo o principal dispositivo para ventilação durante a reanimação cardiopulmonar ${ }^{(2)}$. Também fornece oxigênio aos pacientes, substitui temporariamente a ventilação mecânica ${ }^{(3)}$, ventila pacientes durante o transporte intra e extra-hospitalar e é utilizado na fisioterapia respiratória ${ }^{(4)}$.

Equipamentos de ventilação artificial portáteis só passaram a ser utilizados rotineiramente em 1952, durante epidemia de poliomielite. A partir dessa época, houve desenvolvimento acelerado dos $\mathrm{RM}^{(5)}$. Em 1955, a empresa Ambu International inventou o primeiro RM portátil autoinflável, com válvula de não reinalação, que recebeu o nome de $\mathrm{Ambu}^{\circledR(6)}$. O $\mathrm{Ambu}^{\circledR}$ passou a ser usado como sinônimo de RM em todo o mundo. Novos equipamentos foram aprimorados para garantir segurança ao paciente e praticidade ao profissional que o manipula ${ }^{(7)}$.
Atualmente, existem três tipos de RM: autoinflável, inflado por fluxo e em peça T. A diferença entre eles é funcionamento ${ }^{(8)}$. Os reanimadores inflados por fluxo e em peça $T$ necessitam receber fluxo contínuo de gás ou precisam estar conectados à fonte elétrica, já o RM autoinflável se autoexpande após ter a bolsa descomprimida. A grande vantagem do RM é ser portátil e de fácil utilização ${ }^{(9)}$. Nesta revisão, serão analisados apenas os estudos sobre os RM autoinfláveis.

Um RM possui quatro partes: porta expiratória, válvula reguladora de pressão, bolsa e válvula de entrada de ar. Componentes opcionais são: reservatório de oxigênio, válvula de pressão expiratória positiva final (PEEP) e manômetro ${ }^{(10,11)}$ (Figura 1).

A porta expiratória contém a válvula de não reinalação (válvula do paciente). É unidirecional e permite fluxo de ar para o paciente quando a bolsa é comprimida e se fecha quando ocorre liberação da bolsa, evitando a entrada de ar retrógrado, pois não permite que o gás exalado seja reinalado durante a próxima insuflação.

A válvula limitadora de pressão ou válvula pop-off é utilizada com a finalidade de adequar o pico de pressão (PIP) atingido pelo RM. A American Society for Testing and Materials (ASTM) preconiza que todos os RM possuam essa válvula e que haja um alívio da pressão ao se atingir PIP de $40 \pm 5 \mathrm{cmH}_{2} \mathrm{O}^{(12)}$. A maioria dos aparelhos para neonatos e crianças é equipada com essa válvula e, caso o profissional necessite empregar picos superiores, a válvula pode ser bloqueada.

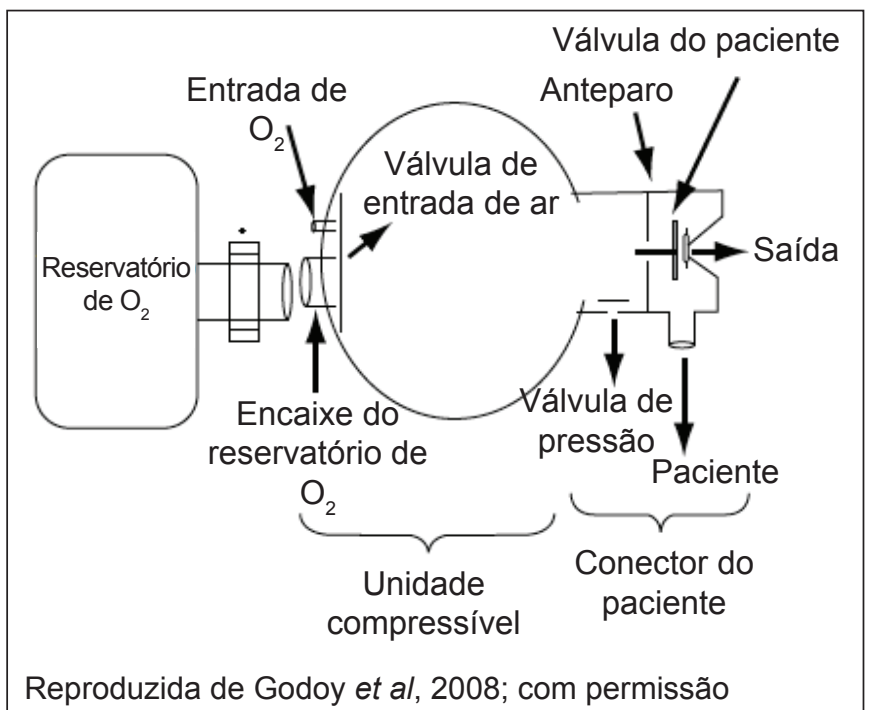

Figura 1 - Representação esquemática dos componentes do reanimador manual(11). 
A bolsa é a unidade compressível e deve se expandir após cada compressão. A frequência de reinsuflação da bolsa determina o volume minuto máximo. A manutenção da compressão da bolsa durante a fase expiratória reduz o pico de fluxo expiratório, aumentando a relação inspiração/expiração ${ }^{(13)}$.

A válvula de entrada de ar evita a saída deste pela base da bolsa quando o ressuscitador é comprimido. É responsável pela entrada do ar ambiente na bolsa, quando a pressão no interior desta é liberada.

O reservatório de oxigênio aumenta a fração do mesmo $\left(\mathrm{FiO}_{2}\right)$ fornecida ao paciente. Alguns aparelhos permitem que essa bolsa, na forma de saco, seja acoplada à válvula de entrada de ar na base da bolsa. Para o funcionamento desse dispositivo, o ressuscitador precisa ser alimentado por uma fonte de oxigênio. Um fluxo adequado deve ser ajustado para a expansão completa do reservatório, sem que ele colapse durante o enchimento da bolsa. Quando uma fonte suplementar de oxigênio não é utilizada, o reservatório deve ser removido, pois pode afetar o enchimento da bolsa ${ }^{(14)}$. Existem duas válvulas no reservatório. Uma válvula safety outlet alivia a pressão no interior do reservatório caso um fluxo de ar muito alto seja fornecido ou não haja manipulação da bolsa. Se o volume de gás no reservatório é insuficiente para encher a bolsa, outra válvula safety inlet possibilita a entrada de ar ambiente.

A válvula de PEEP fornece PEEP e pode ser acoplada na porta expiratória do RM. Ela evita o desrecrutamento alveolar durante a ventilação manual ${ }^{(15)}$.

O manômetro é usado para monitorar a PIP gerada durante a ventilação manual. Seu emprego acoplado ao RM foi testado em dois estudos ${ }^{(16,17)}$. Ambos indicaram que $\mathrm{o}$ manômetro aumenta a acurácia e reduz a variabilidade do desempenho da ventilação. Por isso, é recomendado seu uso na prática clínica.

A compressão do RM provoca aumento da pressão interna da bolsa e bloqueia a válvula de entrada de ar, forçando a abertura da válvula do paciente sobre a porta expiratória. Com a descompressão, a pressão na válvula do paciente é removida e se fecha. A liberação da pressão no interior da bolsa abre a válvula de entrada de ar, permitindo entrada de gás para a próxima insuflação ${ }^{(18)}$.

O manuseio do RM deveria ser restrito a pessoas qualificadas e treinadas $^{(14)}$. Compressões inadequadas podem causar hipoventilação, hiperventilação, barotrauma e redução do débito cardíaco. O RM deve ser utilizado com a válvula reguladora de pressão desbloqueada ${ }^{(14)} \mathrm{e}$ a pressão do aparelho monitorada com manômetro ${ }^{(16,17)}$.
A válvula de não reinalação do RM pode se contaminar com vômito, sangue ou secreção durante a ventilação. Por isso, antes de conectar o reanimador no tubo endotraqueal, é preciso checar o equipamento. Deve-se testar o funcionamento do aparelho para não haver reinalação de gás carbônico $\left(\mathrm{CO}_{2}\right)^{(14)}$.

Embora amplamente utilizado e comercializado em todo o mundo, existe pouca informação disponível sobre as características funcionais e físicas dos RM. Apesar da padronização do RM pela ASTM ${ }^{(12)}$, estudos mostram que diversos fatores afetam os parâmetros gerados por esses aparelhos. Poucos estudos sistematizados e bem controlados analisaram e compararam os RM. Dentre os poucos existentes, os resultados são conflitantes e não existe revisão de literatura sobre o assunto. É fundamental que os profissionais da área da saúde conheçam as diferenças entre os $\mathrm{RM}$ e os fatores que interferem nos desfechos ventilatórios com a utilização dos mesmos.

$\mathrm{O}$ objetivo desse estudo foi verificar as evidências dos fatores que influenciam a ventilação pulmonar com os RM infantis e adultos por meio de uma revisão sistemática da literatura.

\section{Método}

Realizou-se uma revisão sistemática da literatura, buscando artigos originais indexados nas bases de dados Medline, Lilacs e SciELO publicados entre Janeiro de 1986 e Março de 2011. As palavras-chave utilizadas na busca eletrônica foram: "reanimador manual", "ressuscitador manual" ("manual resuscitator"), "ventilação manual" ("manual ventilation"), "ventilação com pressão positiva" ("positive pressure ventilation") e "bag-valve”.

Foram encontrados 69 artigos, 63 em inglês e seis em português. Incluíram-se 56 trabalhos que compararam os RM por marcas, modelos e analisaram as características dos profissionais que manuseavam os mesmos, estudos que avaliaram o funcionamento do RM em diversas situações experimentais e clínicas. Aqueles que analisaram unicamente os reanimadores mecânicos foram excluídos. Duas pesquisadoras analisaram de forma independente os estudos para verificar a concordância, garantindo a reprodutibilidade e a confiabilidade.

\section{Fatores que interferem nos desfechos da ventilação pulmonar com os RM}

A maior parte dos artigos encontrados sobre os RM adulto e pediátrico são experimentais. A referência dos principais artigos encontrados, seus objetivos e conclusões encontram- 
se resumidos de acordo com o modelo de RM estudado -se adulto (Quadro 1) ou se pediátrico (Quadro 2).

Os principais fatores de influência nos desfechos da ventilação com RM encontrados a partir dessa revisão sistemática de literatura são: forma de manuseio do reanimador manual, formação profissional do operador do reanimador, treinamento e experiência profissional, marcas e modelos do reanimador manual, válvula limitadora de pressão do reanimador e fluxo de oxigênio fornecido ao reanimador.

\section{Forma de manuseio dos RM em adultos}

Alguns estudos compararam diferentes técnicas de ventilação pulmonar, formas de manipulação e influências dos profissionais que operam o $\mathrm{RM}^{(19-23)}$.

Quadro 1 - Resumo dos principais artigos sobre reanimador manual modelo adulto

\begin{tabular}{|c|c|c|}
\hline Referência & Objetivo & Conclusão \\
\hline Hess e Spahr $r^{(21)}$ & $\begin{array}{l}\text { Verificar efeito do tamanho da } \\
\text { mão, uso de luvas e número de } \\
\text { mãos no VC fornecido por } 5 \text { RMs } \\
\text { descartáveis }\end{array}$ & $\begin{array}{l}\text { O VC aumentou de acordo com aumento do tamanho } \\
\text { da mão e o número de mãos usadas na compressão. } \\
\text { Foi observada diferença no VC entre marcas. Uso de } \\
\text { luvas não afetou VC }\end{array}$ \\
\hline Shawn e Suzanne ${ }^{(19)}$ & $\begin{array}{l}\text { Comparar efeito da compressão } \\
\text { do RM com } 1 \text { e } 2 \text { mãos e das } \\
\text { características dos operadores no } \\
\text { VC fornecido }\end{array}$ & $\begin{array}{l}\text { O VC fornecido utilizando } 2 \text { mãos foi maior do que } \\
\text { com } 1 \text { mão. A força de preensão manual foi o melhor } \\
\text { preditor do VC e apresentou maior correlação com o } \\
\text { VC fornecido com } 1 \text { mão }\end{array}$ \\
\hline Lee et $a)^{(22)}$ & $\begin{array}{l}\text { Comparar método de compressão } \\
\text { do RM, características dos } \\
\text { operadores e nível de experiência } \\
\text { no VC fornecido }\end{array}$ & $\begin{array}{l}\text { O VC gerado com } 2 \text { mãos foi maior do que com } 1 \\
\text { mão. O tamanho da mão, volume e força não se } \\
\text { associaram ao VC fornecido }\end{array}$ \\
\hline Barnes e McGarry ${ }^{(42)}$ & $\begin{array}{l}\text { Avaliar desempenho e segurança } \\
\text { de } 10 \text { RMs descartáveis ( } 6 \\
\text { adultos e } 4 \text { pediátricos) }\end{array}$ & $\begin{array}{l}\text { Apenas o Code Blue }{ }^{\otimes}, 1^{\text {st }} \text { response }{ }^{\otimes} \text {, Pulmanex }{ }^{\circledR} \text { e o } \\
\text { SPUR }^{\circledR} \text {, dentre os testados, seguiam as normas da } \\
\text { ASTM F- } 920 \text { e podem substituir os RMs reusáveis }\end{array}$ \\
\hline Kissoon et $a /^{(20)}$ & $\begin{array}{l}\text { Avaliar desempenho de } 8 \text { RMs } \\
\text { descartáveis }\end{array}$ & $\begin{array}{l}\text { Apesar dos RMs testados estarem de acordo com a } \\
\text { padronização mínima, estes devem ser escolhidos de } \\
\text { forma cuidadosa e avaliados antes do uso clínico }\end{array}$ \\
\hline Barnes e Stockwell(43) & $\begin{array}{l}\text { Avaliar desempenho e segurança } \\
\text { de } 10 \text { RMs ( } 5 \text { reusáveis e } 5 \\
\text { descartáveis) }\end{array}$ & $\begin{array}{l}\text { Apenas o Code } \text { Blue }^{\otimes} \text { e DMR } \\
\text { seguiam as normas da ASTM e ISO na temperatura } \\
\text { operacional de }-18 \text { a } 50^{\circ} \mathrm{C}\end{array}$ \\
\hline Godoy et al(11) & $\begin{array}{l}\text { Determinar alterações do VC e } \\
\text { PIP fornecidos por } 7 \text { modelos } \\
\text { de RMs em função do fluxo de } \\
\text { entrada de } \mathrm{O}_{2}\end{array}$ & $\begin{array}{l}\text { Em RMs com } \mathrm{O}_{2} \text { fornecido diretamente no interior do } \\
\text { balão, se receberem fluxos } \geq 5 \mathrm{~L} / \mathrm{min} \text {, há elevação do } \\
\text { VC e PIP }\end{array}$ \\
\hline Godoy et a/(18) & $\begin{array}{l}\text { Determinar fluxo de } \mathrm{O}_{2} \text { fornecido } \\
\text { por } 7 \text { marcas de } \mathrm{RM} \text { (com e sem } \\
\text { reservatório de } \mathrm{O}_{2} \text { ) }\end{array}$ & $\begin{array}{l}\text { O RM que permite acoplar reservatório de } \mathrm{O}_{2} \text { deve } \\
\text { obrigatoriamente usá-lo quando aplicado como fonte } \\
\text { de } \mathrm{O}_{2} \text { nos pacientes em respiração espontânea. } \\
\text { Todos os modelos estudados devem receber fluxo de } \\
\mathrm{O}_{2}>15 \mathrm{~L} / \mathrm{min}\end{array}$ \\
\hline Mills et $a /^{(1)}$ & $\begin{array}{l}\text { Comparar } \mathrm{FiO}_{2} \text { fornecida por } \\
13 \mathrm{RMs} \text { para pacientes em } \\
\text { ventilação espontânea }\end{array}$ & $\begin{array}{l}\text { O RM administra alta } \mathrm{FiO}_{2} \text { e permite ventilar os } \\
\text { pacientes manualmente. Esse resultado é melhor } \\
\text { alcançado com RMs que possuem válvula de baixa } \\
\text { resistência a disco }\end{array}$ \\
\hline Godoy et al(52) & $\begin{array}{l}\text { Determinar a } \mathrm{FiO}_{2} \text { ofertada por } 7 \\
\text { RMs em função do fluxo de } \mathrm{O}_{2}\end{array}$ & $\begin{array}{l}\text { Os RMs com opção de acoplar o reservatório } \\
\text { fornecem maior } \mathrm{FiO}_{2} \text { com esse acessório; já os } \\
\text { que não apresentam a possibilidade de acoplá- } \\
\text { lo apresentam maior } \mathrm{FiO}_{2} \text { em relação aos demais } \\
\text { quando usados sem esse acessório }\end{array}$ \\
\hline
\end{tabular}

VC: volume corrente; RM: reanimador manual; ASTM: American Society for Testing and Materials; ISO: International Organization for Standardization; PIP: pico de pressão inspiratório; $\mathrm{O}_{2}$ : oxigênio; $\mathrm{FiO}_{2}$ : fração inspirada de oxigênio. 
Quadro 2 - Resumo dos principais artigos sobre reanimador manual modelo pediátrico

\begin{tabular}{|c|c|c|}
\hline Referência & Objetivo & Conclusão \\
\hline Resende et $a /^{(37)}$ & $\begin{array}{l}\text { Avaliar PIP e frequência } \\
\text { ventilatória obtidos por médicos } \\
\text { utilizando o RM em modelo de } \\
\text { pulmão }\end{array}$ & $\begin{array}{l}\text { A ventilação teve frequência adequada em quase } 80 \% \text { das } \\
\text { vezes. Os médicos não conseguiram promover ventilação com } \\
\text { mínima variabilidade na PIP, alcançando níveis diferentes dos } \\
\text { definidos para RCP neonatal em } 70 \% \text { das vezes }\end{array}$ \\
\hline Bassani et al(24) & $\begin{array}{l}\text { Avaliar a influência do } \\
\text { manuseio do RM sobre a PIP } \\
\text { e VC }\end{array}$ & $\begin{array}{l}\text { A maioria dos participantes gerou PIP e VC altos, } \\
\text { principalmente usando } 2 \text { mãos para comprimir o RM. Não foi } \\
\text { detectada influência da profissão sobre as variáveis estudadas }\end{array}$ \\
\hline Field et a/(45) & Avaliar 5 RMs em bebês & $\begin{array}{l}\text { O RM Laerdal }{ }^{\circledR} \text { neonatal e o Penlon }{ }^{\circledR} \text { forneceram baixo VC, não } \\
\text { sendo adequados para uso em RCP. Os RMs com maior bolsa, } \\
\text { Laerdal }^{\circledR} \text { pediátrico e Ambu }{ }^{\circledR} \text {, forneceram maior VC e tempo de } \\
\text { insuflação. }\end{array}$ \\
\hline Finer et $a^{(46)}$ & $\begin{array}{l}\text { Caracterizar o desempenho de } \\
\text { RMs de } 3 \text { marcas }\end{array}$ & $\begin{array}{l}\mathrm{FiO}_{2} \text { foi muito maior com o reservatório acoplado e a ativação } \\
\text { da válvula de pressão reduziu a } \mathrm{FiO}_{2} \text {. A pressão de ativações } \\
\text { da válvula variou muito. } \mathrm{O} \text { Laerdal }{ }^{\otimes} \text { obteve maiores } \mathrm{FiO}_{2}\end{array}$ \\
\hline Connors et al(47) & $\begin{array}{l}\text { Comparar características } \\
\text { físicas e funcionais de } 5 \text { RMs }\end{array}$ & $\begin{array}{l}\text { Alguns RMs comercialmente disponíveis não estão de } \\
\text { acordo com as normas da ASTM e podem não ser ideais em } \\
\text { determinadas situações clínicas }\end{array}$ \\
\hline Hussey et $a^{\left({ }^{(9)}\right.}$ & $\begin{array}{l}\text { Comparar } 3 \text { dispositivos de } \\
\text { ventilação manual }\end{array}$ & $\begin{array}{l}\text { A bolsa anestésica + manômetro e o Neopuff }{ }^{\circledR} \text { facilitaram a } \\
\text { ventilação precisa. O RM deve ser acoplado a um manômetro e } \\
\text { válvula de PEEP, principalmente na RCP do RN prematuro }\end{array}$ \\
\hline Finer et $a^{(26)}$ & $\begin{array}{l}\text { Comparar dispositivos } \\
\text { para ventilação manual e a } \\
\text { capacidade de diferentes } \\
\text { operadores para administrar } \\
\text { PIP e PEEP predefinida }\end{array}$ & $\begin{array}{l}\text { As PIPs e PEEPs fornecidas variaram entre os operadores } \\
\text { com qualquer dispositivo, sendo os maiores valores obtidos } \\
\text { pelos terapeutas respiratórios. O Neopuff }{ }^{\circledR} \text { obteve pressões } \\
\text { independentes do operador e facilitou manutenção das } \\
\text { insuflações }\end{array}$ \\
\hline Bennett et $a l^{(8)}$ & $\begin{array}{l}\text { Comparar habilidade de ofertar } \\
\text { ventilação predeterminada com } \\
3 \text { dispositivos de ventilação } \\
\text { manual }\end{array}$ & $\begin{array}{l}\text { O ressuscitador em peça T ofertou as pressões desejadas e } \\
\text { foi mais preciso, mas levou maior tempo para aumentar a PIP } \\
\text { de } 20 \text { para } 40 \mathrm{~cm}_{2} \mathrm{O} \text {. Com o RM foi difícil manter insuflação } \\
\text { prolongada e ofertar a PEEP desejada, mesmo com a válvula } \\
\text { de PEEP }\end{array}$ \\
\hline Roehr ${ }^{(38)}$ & $\begin{array}{l}\text { Comparar dispositivos } \\
\text { de ventilação manual e } \\
\text { experiência profissional na } \\
\text { RCP simulada }\end{array}$ & $\begin{array}{l}\text { O T-piece garante VT e PIP constante, independente da } \\
\text { característica do operador. O RM obteve VT e PIP maiores, } \\
\text { além de maior variabilidade intersujeitos. A experiência não } \\
\text { influenciou no valor e variabilidade do VT e PIP fornecidos }\end{array}$ \\
\hline Dawson et al ${ }^{(33)}$ & $\begin{array}{l}\text { Comparar capacidade de } 3 \\
\text { dispositivos de ventilação para } \\
\text { fornecer PIP e PEEP }\end{array}$ & $\begin{array}{l}\text { O ressuscitador em peça T foi acurado e consistente. A } \\
\text { bolsa anestésica apresentou maior variação na ventilação. } \\
\text { O vazamento de ar na máscara e o PEEP foram menores } \\
\text { com o RM. Porém houve alta variabilidade com todos os } \\
\text { equipamentos }\end{array}$ \\
\hline Kattwinkel et a/(53) & $\begin{array}{l}\text { Testar habilidade do } \\
\text { profissional em perceber } \\
\text { alterações na complacência } \\
\text { utilizando RM, bolsa anestésica } \\
\text { ou ressuscitador em peça T }\end{array}$ & $\begin{array}{l}\text { A visualização do VC permite melhor detecção de mudanças } \\
\text { na complacência do que somente a PIP. Os aparelhos de } \\
\text { ventilação deveriam mostrar o VC empregado }\end{array}$ \\
\hline
\end{tabular}

PIP: pico de pressão inspiratório; RM: reanimador manual; RCP: ressuscitação cardiopulmonar; , VC: volume corrente; $\mathrm{FiO}_{2}$ : fração inspirada de oxigênio; ASTM: American Society for Testing and Materials; PEEP: pressão expiratória positiva final; RN: recém-nascido. 
Shawm e Suzanne pesquisaram 108 profissionais da saúde utilizando os RM modelo adulto. Verificaram que o volume corrente (VC) empregado ao comprimir a bolsa com duas mãos foi maior do que com uma mão. Houve correlação positiva entre o tamanho da mão, a força de preensão manual, a altura e o peso dos profissionais com o VC fornecido ${ }^{(19)}$. Kissoon et al ${ }^{(20)}$ e Hess e Spahr ${ }^{(21)}$ também observaram aumento do VC fornecido quando as duas mãos comprimiam o RM. Hess e Spahr ${ }^{(21)}$ verificaram o efeito do tamanho da mão nos volumes fornecidos por cinco reanimadores adultos descartáveis e notaram valores mais elevados de VC quando os RM eram operados com mãos maiores.

Por outro lado, os achados de Lee et a ${ }^{(22)}$ e Augustine et al ${ }^{(23)}$ foram parcialmente díspares. O resultado da comparação do número de mãos empregado para comprimir o balão esteve de acordo com a literatura, ou seja, o VC é maior quando duas mãos são utilizadas na compressão. Porém, não houve associação com o tamanho das mãos. No entanto, os tipos de reanimadores utilizados nessas pesquisas foram distintos e houve grande diferença entre os tamanhos amostrais, o que pode justificar as discrepâncias observadas.

Quanto à influência da força de preensão da mão que comprime o balão durante a ventilação, Lee et al ${ }^{(22)} \mathrm{e}$ Shawm e Suzanne ${ }^{(19)}$ obtiveram resultados conflitantes. Esses estudos avaliaram a influência das características físicas dos profissionais no desempenho da ressuscitação e realizaram a dinamometria em 114 e 108 participantes das pesquisas, respectivamente. Enquanto Shawm e Suzanne ${ }^{(19)}$ relataram associação da força manual com o VC gerado pelo RM, Lee et $a^{(22)}$ não obtiveram o mesmo resultado. Shawm e Suzanne ${ }^{(19)}$ ainda descreveram maior relação do VC com a força manual quando usada apenas uma mão, em vez de duas.

Turki et al $^{(3)}$, ao pesquisarem o PIP fornecido com o RM, encontraram diferenças nos desfechos entre profissionais de sexos distintos, sugerindo que os profissionais do sexo masculino, por possuírem maior força muscular, geram uma PIP maior. Entretanto, não foi realizada nenhuma avaliação da força de preensão dos participantes nesse estudo.

\section{Forma de manuseio dos RM em recém-nascidos}

Bassani et al ${ }^{(24)}$ observaram que o modo de manuseio do RM influenciou significativamente o PIP e o VC apenas quando o balão autoinflável foi comprimido com as duas mãos. Segundo esses autores, o VC e o PIP apresentam valores mais altos quanto maior a quantidade de dedos usados. Ganga-Zandzou et al ${ }^{(25)}$ encontraram diferença apenas entre o PIP gerado pela ventilação com cinco e dois dedos. É possível que a diferença entre esses estudos não se deva somente à diferença dos manuseios testados, mas também ao tamanho amostral: no primeiro estudo 141 pacientes foram avaliados e o segundo contou com apenas 19 participantes. Porém, há consenso de que o valor do PIP tende a ser maior quanto mais dedos são usados na compressão da bolsa ${ }^{(24,25)}$.

Existe falta de evidência na literatura pediátrica quanto à influência da força de preensão e do tamanho da mão do profissional na ventilação com o RM.

\section{Formação profissional}

A ventilação adequada é essencial para uma ressuscitação cardiopulmonar bem-sucedida. A forma de suporte ventilatório mais comum em emergências é por meio do RM. Diferentes profissionais de nível superior da área da saúde e alguns técnicos treinados utilizam o reanimador para ventilação em unidades de terapia intensiva, prontosocorros e fora do hospital. Poucas pesquisas compararam o desempenho da ventilação entre profissionais de diversas formações.

Em adultos, Augustine et a $l^{(23)}$ avaliaram o manuseio do equipamento por diferentes operadores da área da saúde e encontraram grande variação nos desfechos ventilatórios entre os profissionais. Constataram que as enfermeiras forneceram os maiores $\mathrm{VC}$ e os médicos, os menores. Os paramédicos geraram os maiores PIP, enquanto os terapeutas respiratórios os menores, porém, a maioria desses profissionais empregou um volume adequado e uma pressão segura durante a ventilação.

Finer et al ${ }^{(26)}$ compararam a reanimação manual de recém-nascidos realizada por vários profissionais em um manequim. Houve diferença significativa entre os 27 profissionais estudados: cinco residentes pediátricos, cinco enfermeiros, quatro médicos neonatologistas, seis residentes neonatais, dois técnicos de enfermagem e cinco terapeutas respiratórios. Os fisioterapeutas forneceram maiores pressões do que os demais profissionais.

Ainda em neonatos, Bassani et $a^{(24)}$ avaliaram a influência do manuseio do reanimador neonatal por diversos profissionais e não detectaram influência da profissão sobre o VC e PIP. Hussey et a ${ }^{(9)}$ também não encontraram diferença nas pressões obtidas por diferentes profissionais. Além disso, a frequência de ventilação não foi dependente da formação profissional, quando comparados médicos e outros profissionais. 
Zaconeta et al $^{(27)}$ avaliaram o PIP e a frequência respiratória (FR) fornecidos por socorristas do Corpo de Bombeiros utilizando o RM em modelo de pulmão neonatal pré-termo. Os socorristas, na maioria das vezes, não atingiram o nível mínimo desejado de PIP $\left(20 \mathrm{cmH}_{2} \mathrm{O}\right)$ e FR ( 40 ciclos/min), porém $64,5 \%$ não tinham experiência em ventilação neonatal. $\mathrm{O}$ autor sugere ter havido maior precaução por parte dos socorristas que, embora tenham recebido instrução sobre reanimação neonatal, nunca haviam realizado o procedimento em uma situação real.

\section{Treinamento profissional}

O treinamento de profissionais para realizar a reanimação cardiorrespiratória efetiva inclui conhecimento e prática na utilização do RM. Existem diversas formas de treinamento: cursos informais, simulação em manequim, treinamento na beira do leito e cursos formais fornecidos por centros de treinamento como a American Heart Association (Basic Life Support, Advanced Life Support e Pediatric Advanced Life Support ${ }^{(28)}$. Segundo Moser e Coleman ${ }^{(29)}$ e Berden et al ${ }^{(30)}$, o retreinamento é útil e necessário, pois a retenção das habilidades de ressuscitação é pequena. Eles estudaram o suporte de vida em adultos e avaliaram o desempenho do Advanced Life Support entre diferentes especialidades médicas. De acordo com os autores, a habilidade dos profissionais é baseada na quantidade de treinamento inerente à sua prática. Sem o reforço do treinamento, a maioria dos médicos perde suas habilidades entre seis e 12 meses após o treinamento.

A ventilação manual é uma habilidade que requer ensino e aprendizagem teórico-prático ${ }^{(31)}$. Uma ventilação acurada é necessária, principalmente em situações de emergência, pois tanto a hiperventilação, quanto a hipoventilação, podem ser iatrogênicas. Lee et a ${ }^{(22)}$ descrevem cinco etapas do nível de experiência: conhecimento do suporte básico de vida, dos reanimadores manuais, treinamento e experiência com o RM em manequim, experiência com pacientes e experiência em ressuscitação cardiopulmonar. Os autores compararam o VC fornecido por operadores do aparelho com diferentes níveis de treinamento e encontraram discreto aumento do VC em modelos do tipo adulto de acordo com o nível de experiência; entretanto, essa correlação foi fraca. Como apenas esse estudo abordou o efeito do ensino da técnica de reanimação manual na ressuscitação cardiopulmonar, permanece incerta a associação da prática clínica com o desempenho da ventilação.
Diversos estudos avaliando dispositivos para reanimação neonatal confirmam a importância do treinamento para a utilização do $\mathrm{RM}^{(26,32-36)}$. Apenas o estudo de Resende $e t$ al ${ }^{(37)}$, realizado com médicos em modelo de pulmão neonatal, questiona a afirmação de que há necessidade de prática para que o profissional tenha êxito na reanimação, pois, segundo o autor, apesar de os médicos serem capacitados, a ventilação não foi adequada. Entretanto o pesquisador não especifica a frequência com que esses profissionais praticam a reanimação cardiopulmonar.

Roehr $e t$ al ${ }^{(38)}$ estudaram o efeito da experiência profissional no VC e PIP aplicados na ressuscitação simulada em manequim neonatal. Os pesquisadores compararam diferentes dispositivos de ventilação manual utilizando 120 profissionais. Não foi encontrada influência da experiência no valor e na variabilidade do VC e PIP fornecidos.

Não foram recuperados artigos que avaliassem o treinamento da ventilação manual em pediatria. Esses estudos seriam de grande valia, já que a ressuscitação pediátrica difere da adulta em diversos aspectos. A primeira depende da identificação e tratamento dos eventos respiratórios antes dos cardíacos, ao contrário do que ocorre na maioria dos eventos de reanimação de adultos. Outro aspecto é a menor frequência da ressuscitação pediátrica comparada com a reanimação em adultos. Os cursos de treinamento para suporte de vida em adultos são diferentes daqueles feitos com pacientes pediátricos ${ }^{(39)}$.

\section{Marcas e modelos de reanimador}

Ainda que os consensos internacionais definam o RM como o principal instrumento para ventilação manual, estudos têm mostrado que não há unanimidade quanto aos equipamentos utilizados na reanimação ${ }^{(40,41)}$. Existe grande diversidade de marcas/modelos disponíveis comercialmente utilizados.

Pesquisadores ${ }^{(42,43)}$ avaliaram o desempenho e a segurança de diversos balões autoinfláveis em um pulmão-teste. Um pesquisador estudou seis marcas de RM descartáveis adultos e quatro marcas pediátricas. Outro utilizou em temperaturas extremas (18 a $50^{\circ} \mathrm{C}$ ) em cinco aparelhos descartáveis e cinco reutilizáveis. Quase todos os testes com ressuscitadores pediátricos ultrapassaram PIP de $40 \pm 10 \mathrm{cmH}_{2} \mathrm{O}$, representando alto risco de barotrauma. A maioria dos reanimadores sob temperaturas muito baixas não seguiu as normas da ASTM, apresentando $\mathrm{FiO}_{2}$ inadequada e não passando no teste de queda de $1 \mathrm{~m}$ de altura. De acordo com a pesquisa de Barnes e McGarry ${ }^{(42)}$, apenas três marcas (Code Blue ${ }^{\text {st }}$ response ${ }^{\circledR}$, Pulmanex ${ }^{\circledR}$ e SPUR $^{\circledR}$ ) estavam de acordo com as normas da ASTM F-920. 
Ao compararem o desempenho de cinco marcas de reanimadores adultos descartáveis, Hess e Spahr ${ }^{(21)}$ sugerem que, em alguns casos, a diferença de volumes ofertados pelas marcas pode ser clinicamente importante. Kissoon et al ${ }^{(20)}$ avaliaram oito marcas de reanimadores descartáveis e verificaram que as marcas $\mathrm{CPR}^{\circledR}$ e $\mathrm{LSP}^{\circledR}$ podem sofrer erro de montagem. Outras marcas $\left(\mathrm{B}-\mathrm{Sav}^{\circledR}, \mathrm{Pulm}^{\circledR}\right.$ e $\mathrm{MPR}^{\circledR}$ ) forneceram $\mathrm{FiO}_{2}<80 \%$.

Mazzolini Jr e Marshall ${ }^{(44)}$ realizaram teste comparativo de reanimadores adultos descartáveis de nove marcas e 16 modelos, seguindo normas da ASTM e da International Organization for Standardization (ISO). Avaliaram dados referentes ao desenho do reservatório, facilidade de acoplar a válvula de PEEP, tamanho do aparelho, textura, presença do detector de $\mathrm{CO}_{2}$, tipo de material, $\mathrm{FiO}_{2}, \mathrm{VC}$, teste de queda e bloqueio da válvula do paciente. Os resultados encontrados demonstram que o tipo e o desenho do reservatório afeta a $\mathrm{FiO}_{2}$ fornecida pelos equipamentos. Os reservatórios em formato de bolsa fornecem maior $\mathrm{FiO}_{2}$ do que os em forma de tubo. Todos os modelos passaram no teste de queda da altura de $1 \mathrm{~m}$ e tiveram VC aceitáveis. A maioria tinha tamanho médio e boa textura, porém alguns eram grandes, escorregadios e difíceis de manusear. Nenhum deles teve a válvula do paciente bloqueada com altos fluxos de entrada. Somente dois tinham detector de $\mathrm{CO}_{2}$ acoplado e apenas em um reanimador houve facilidade de acoplar a válvula de PEEP de forma segura.

A fração de oxigênio fornecida por 13 reanimadores para pacientes adultos em ventilação espontânea foi testada por Mills et $a l^{(1)}$. Foram analisadas: fonte de $\mathrm{O}_{2}$, tipo de válvula, marcas, frequência de ventilação, $\mathrm{VC}$ e pressão resistiva. Concluiu-se que fonte de $\mathrm{O}_{2}, \mathrm{VC}$, ventilação-minuto e volume do reservatório interferem na $\mathrm{FiO}_{2}$, porém o fator mais importante é o tipo da válvula do paciente. As válvulas a disco, que previnem a entrada de ar pela porta expiratória, forneceram $\mathrm{O}_{2}$ de forma mais eficiente, enquanto válvulas "bico-de-pato" não preveniram a entrada de ar. Apenas dois aparelhos mostraram alta pressão resistiva. Os autores concluíram que, para administrar $\mathrm{FiO}_{2}$ elevada para pacientes em ventilação espontânea, são necessários aparelhos com válvula de baixa resistência à disco.

Godoy et $a^{(18)}$ testaram o fluxo de $\mathrm{O}_{2}$ fornecido por sete marcas de reanimadores, com e sem o reservatório, simulando o uso do aparelho adulto em pacientes em respiração espontânea como fonte de $\mathrm{O}_{2}$. Utilizaram fluxos de entrada de $\mathrm{O}_{2}$ de 1 , 5, 10 e $15 \mathrm{~L} / \mathrm{min}$. Concluíram que todas as marcas forneceram maior fluxo de saída quando receberam $15 \mathrm{~L} / \mathrm{min}$ de $\mathrm{O}_{2}$. Os ressuscitadores apresentaram maior fluxo de saída quando o reservatório de $\mathrm{O}_{2}$ estava acoplado. Contudo, mesmo com o uso do reservatório e $15 \mathrm{~L} / \mathrm{min}$, nem todas as marcas forneceram fluxo de saída de $\mathrm{O}_{2}$ suficiente.

Outro estudo do mesmo pesquisador ${ }^{(11)}$ discutiu a influência de marcas diferentes no uso do equipamento em pacientes adultos, determinando alterações do VC e PIP de acordo com o fluxo de entrada de $\mathrm{O}_{2}$. Apenas os modelos Oxigel- $\mathrm{A}^{\circledR}$ e Narcosul ${ }^{\circledR}$ forneceram VC e PIP superiores com fluxo de $15 \mathrm{~L} /$ minuto de $\mathrm{O}_{2}$ do que quando receberam $1 \mathrm{~L} / \mathrm{min}$. Segundo o autor, nos aparelhos com fluxo de $\mathrm{O}_{2}$ direcionado no interior da bolsa, houve bloqueio da válvula do paciente ao utilizar fluxos acima de $5 \mathrm{~L} / \mathrm{min}$, aumentando o VC e o PIP fornecidos. Uma possível limitação do estudo é a de que a manipulação do RM foi realizada por um único indivíduo durante a pesquisa, não sendo este cego quanto aos resultados esperados.

Field $e t$ al ${ }^{(45)}$ avaliaram a efetividade de cinco RM neonatais e pediátricos em dez bebês em apneia após o parto. Relataram que os ressuscitadores com bolsas maiores, como o Laerdal ${ }^{\circledast}$ e Ambu ${ }^{\circledR}$, tiveram maior tempo de insuflação, produzindo maior VC. Já os reanimadores de menor tamanho não forneceram volumes suficientes para a ressuscitação cardiopulmonar. Limitações como pequeno tamanho amostral, exemplar único testado e realização de poucas insuflações podem ter interferido nos resultados.

Em estudo experimental, Finer et al ${ }^{(46)}$ caracterizaram três exemplares de três marcas (Ohio HopeII ${ }^{\circledast}$, PMR $-2^{\circledR} \mathrm{e}$ Laerdal $^{\circledast}$ ) em um pulmão-teste simulando diversas situações. Encontraram ampla variação da pressão de ativação da válvula limitadora: desde $41 \mathrm{cmH}_{2} \mathrm{O}$, no Laerdal ${ }^{\circledR}$, até $106 \mathrm{cmH}_{2} \mathrm{O}$, no Ohio-HopeII ${ }^{\circledR}$. Nesse trabalho também se observou que a $\mathrm{FiO}_{2}$ foi maior com o uso do reservatório, e a ativação da válvula limitadora de pressão contribuiu para reduzir a $\mathrm{FiO}_{2}$.

Connors et $a l^{(47)}$ estudaram as características físicas e funcionais de cinco RM neonatais, verificando a adequação dos mesmos às padronizações da ASTM. De acordo com os pesquisadores, algumas marcas não estavam de acordo com as normas, apresentando diversos problemas, como a ausência de válvula limitadora de pressão, o conector que se soltava facilmente e a válvula expiratória com defeito.

A escolha do RM para situações clínicas deve ser criteriosa. Inicialmente, o RM deve ser testado e, em situações específicas, determinadas marcas podem não ser indicadas ${ }^{(48)}$.

\section{Válvula limitadora de pressão do RM}

$\mathrm{Na}$ prática clínica, os profissionais de saúde não costumam monitorizar as pressões durante a ressuscitação manual e 
utilizam dados subjetivos para avaliar a ventilação do paciente. Costuma-se adequar a pressão avaliando a expansão da caixa torácica e a resistência à ventilação, percebida de forma subjetiva pela mão do operador. Porém, em uma situação de emergência, pode-se não conseguir visualizar a expansibilidade torácica de forma contínua e nem perceber a resistência à insuflação.

Não se recuperaram na literatura, até o presente momento, estudos avaliando a influência da válvula limitadora de pressão nos parâmetros ventilatórios fornecidos pelos RM modelo adulto. Nesses modelos de RM, a válvula não é encontrada em todas as marcas de aparelho. Profissionais não costumam se preocupar com o risco de barotrauma em pacientes adultos.

Em estudos com modelos neonatais, é consenso que a utilização da válvula limitadora de pressão gera variabilidade da pressão de pico empregada durante a ventilação ${ }^{(37,45,47)}$. Finer $e t a l^{(46)}$ demonstraram variabilidade na pressão em que a válvula é ativada, indicando que, algumas vezes, as pressões ultrapassam os valores considerados seguros para o recémnascido $\left(30 \mathrm{cmH}_{2} \mathrm{O}\right)$, embora não exista consenso quanto ao valor ideal de PIP durante a ventilação. Connors et al ${ }^{(47)}$ também sugerem que a pressão de ativação das válvulas não é confiável.

Existem vários tipos de dispositivos para ventilação neonatal e a maioria dos balões autoinfláveis não utiliza manômetro ${ }^{(40)}$. Alguns autores sugerem que o emprego do manômetro acoplado ao reanimador garante um feedback visual e reduz a variabilidade do PIP ofertado pelo aparelho, garantindo maior segurança para os pacientes e reduzindo iatrogenias ${ }^{(16,17)}$.

\section{Fluxo de oxigênio fornecido ao RM}

$\mathrm{O}$ uso de fonte de $\mathrm{O}_{2}$ conectada ao $\mathrm{RM}$ e o local de entrada de oxigênio no balão pode influenciar o PIP e o VC gerados $^{(11,49-51)}$ independentemente da marca do RM.

Em adultos, os reanimadores devem funcionar adequadamente com altos fluxos de $\mathrm{O}_{2}$, pois, durante uma emergência, o fluxômetro pode ser alterado. São relatados incidentes nos quais a válvula do paciente é bloqueada com altos fluxos de oxigênio. Esse prejuízo no funcionamento do reanimador pode colocar o paciente em risco ${ }^{(44)}$.

Godoy et a ${ }^{(52)}$ também determinou que o fluxo de $\mathrm{O}_{2}$ influencia a $\mathrm{FiO}_{2}$ ofertada por sete RMs. Os RMs foram testados utilizando fluxos de $\mathrm{O}_{2}$ de 1, 5, 10 e $15 \mathrm{~L} / \mathrm{min}$ com e sem reservatório de $\mathrm{O}_{2}$. O pesquisador encontrou que os $\mathrm{RM}$ que permitem acoplar o reservatório de $\mathrm{O}_{2}$, quando testados sem esse acessório, atingem $\mathrm{FiO}_{2}$ maior que 0,40 quando recebem fluxo de $\mathrm{O}_{2}$ maior que $10 \mathrm{~L} / \mathrm{min}$. Devido à possibilidade de variação no desempenho dos $\mathrm{RM}$ em função do fluxo de $\mathrm{O}_{2}$ utilizado, os profissionais de saúde devem adequar o fluxo para que o aparelho funcione em segurança ${ }^{(11)}$.

O North American Neonatal Resuscitation Program afirma que o RM pode fornecer ar ambiente, quando nenhuma fonte de gás está conectada. Quando ligado à fonte de $\mathrm{O}_{2} \mathrm{a}$ $100 \%$, recomenda-se que se utilize fluxo de 5 a $10 \mathrm{~L} / \mathrm{min}$ na entrada da bolsa. O RM fornece $\mathrm{FiO}_{2}$ de 80 a $100 \%$ quando o reservatório está conectado e cerca de $40 \%$ de oxigênio, sem reservatório ${ }^{(53)}$.

Diferenças no fluxo de oxigênio fornecido ao RM, a posição da entrada de ar, o tipo de válvula de entrada de ar e o uso do reservatório podem contribuir para a variabilidade da $\mathrm{FiO}_{2}$ dos diferentes ressuscitadores ${ }^{(44,46,54)}$. Thió et al ${ }^{(55)}$ ainda encontraram três principais fatores de influência na $\mathrm{FiO}_{2}$ fornecida por duas marcas de RM neonatais sem reservatório: fluxo de $\mathrm{O}_{2}$, frequência ventilatória e a pressão de pico.

O fluxo de gás parece não interferir na PEEP fornecida pelos $\mathrm{RMs}^{(56)}$. Não foram encontrados na literatura estudos sobre a influência do fluxo de $\mathrm{O}_{2}$ no $\mathrm{VC}$ ou no PIP fornecidos durante a ventilação com reanimadores pediátricos.

\section{Considerações finais}

Estudos mostram que os RM são dispositivos eficazes de ventilação, porém apresentam grande variabilidade nos parâmetros ventilatórios. A formação do profissional que opera o aparelho interfere no desempenho da ventilação, assim como suas características físicas: tamanho da mão, força de preensão manual, quantidade de mãos utilizadas na compressão e modo de manuseio do RM.

A maioria das marcas parece estar de acordo com padrões internacionais de qualidade, contudo, os equipamentos devem ser avaliados antes de sua aplicação no ambiente clínico. Os desfechos ventilatórios, dentre eles a $\mathrm{FiO}_{2}$, sofrem influência do uso de reservatório acoplado, fluxo de entrada de oxigênio, dos tipos de válvula do aparelho e suas características funcionais.

Embora existam diversas pesquisas sobre os RM, são escassos trabalhos sobre modelos utilizados em recémnascidos e crianças. Pesquisas avaliando o desempenho dos $\mathrm{RM}$ neonatal e pediátrico na ventilação pulmonar manual são necessárias. 


\section{Referências bibliográficas}

1. Mills PJ, Baptiste J, Preston J, Barnas GM. Manual resuscitators and spontaneous ventilation--an evaluation. Crit Care Med 1991;19:1425-31.

2. American Heart Association (AHA). Part 6: CPR Techniques and Devices. Circulation 2005;112:IV-47-50.

3. Turki M, Young MP, Wagers SS, Bates JH. Peak Pressures during manual ventilation. Respir Care 2005;50:340-4.

4. MacCarren B, Chow CM. Manual hyperinflation: a description of the technique. Aust J Physiother 1996;42:203-8.

5. Barnes TA. Emergency ventilation techniques and related equipment. Respir Care 1992;37:673-94.

6. Ruben $\mathrm{H}$. Self-contained resuscitation equipment. Can Med Assoc J 1959;80:44-5.

7. Colice GL. Historical perspective on the development of mechanical ventilation. In: Tobin MJ, editor. Principles and practice of mechanical ventilation. $2^{\text {nd }}$ ed. New York: MacGraw-Hill; 2006. p. 1-36.

8. Bennett S, Finer NN, Rich W, Vaucher Y. A comparison of three neonatal resuscitation devices. Resuscitation 2005;67:113-8.

9. Hussey SG, Ryan CA, Murphy BP. Comparison of three manual ventilation devices using an intubated mannequin. Arch Dis Child Fetal Neonatal Ed 2004;89:F490-3.

10. Dorsch JA, Dorsch SE. Manual resuscitators. In: Dorsch JA, Dorsch SE, editors. Understanding anesthesia equipment. $5^{\text {th }}$ ed. Philadelphia: Lippincott Williams \& Wilkins; 2008. p. 282-95.

11. Godoy AC, Vieira RJ, De Capitani EM. Alterations in peak inspiratory pressure and tidal volume delivered by manually operated self-inflating resuscitation bags as a function of the oxygen supply rate. J Bras Pneumol 2008;34:817-21.

12. ASTM International. ASTM Standard F920-93 Standard Specification for Minimum Performance and Safety Requirements for Resuscitators Intended for Use With Humans. West Conshohocken: ASTM International; 1999.

13. Maxwell LJ, Ellis ER. The effect on expiratory flow rate of maintaining bag compression during manual hyperinflation. Aust J Physiother 2004;50:47-9.

14. Hudson $\mathrm{RCI}^{\circledR}$. Lifesaver manual resuscitator-operating manual. Temecula (CA): Hudson RCl ${ }^{\circledR} ; 1993$.

15. Robson WP. To bag or not to bag? Manual hyperinflation in intensive care. Intensive and Crit Care Nurs 1998;14:239-43.

16. Hila J, Ellis E, Holmes W. Feedback withdrawal and changing compliance during manual hyperinflation. Physiother Res Int 2002;7:53-64.

17. Redfern J, Ellis E, Holmes W. The use of a pressure manometer enhances student physiotherapists' performance during manual hyperinflation. Aust $\mathrm{J}$ Physiother 2001;47:121-31.

18. Godoy AC, Vieira RJ, Vieira-Neto RJ. Oxygen outflow delivered by manually operated self-inflating resuscitation bags in patients breathing spontaneously. J Bras Pneumol 2008;34:212-6.

19. Shawn MM, Suzanne C. Comparison of tidal volumes obtained by one-handed and two-handed ventilation techniques. Am J Crit Care 1993;2:467-73.

20. Kissoon N, Nykanen D, Tiffin N, Frewen T, Brasher P. Evaluation of performance characteristics of disposable bag-valve resuscitators. Crit Care Med 1991;19:102-7.

21. Hess D, Spahr C. An evaluation of volumes delivered by selected adult disposable resuscitators: the effects of hand size, number of hands used, and use of disposable medical gloves. Respiratory Care 1990;35:800-5.

22. Lee HM, Cho KH, Choi YH, Yoon SY, Choi YH. Can you deliver accurate tidal volume by manual resuscitator? EMJ 2008;25:632-4.

23. Augustine JA, Seidel DR, McCabe JB. Ventilation performance using a selfinflating anesthesia bag: effect of operator characteristics. Am J Emerg Med 1987:5:267-70.

24. Bassani MA, Filho FM, Coppo MR, Marba ST. Peak pressure and tidal volume are affected by how the neonatal self-inflating bag is handled. J Pediatr 2009;85:217-22

25. Ganga-Zandzou PS, Diependaele JF, Storme L, Riou Y, Klosowski S, Rakza T et al. La ventilation à Ambú ${ }^{\oplus}$ chez nouveau-né: une simple question de doigté? Arch Pediatr 1996;3:1270-2.

26. Finer NN, Rich W, Craft A, Henderson C. Comparison of methods of bag and mask ventilation for neonatal resuscitation. Resuscitation 2001;49:299-305.

27. Zaconeta CAM, Borges MBS, Souza DVB, Marques MG. Avaliação do pico de pressão e da frequência respiratória durante o uso de balão autoinflável por socorristas do Corpo de Bombeiros em um modelo de pulmão neonatal pré-termo. Rev Paul Pediatr 2010;28:5-9.

28. White JR, Shugerman R, Brownlee C, Quan L. Performance of Advanced Resuscitation Skills by Pediatric Housestaff. Arch Pediatr Adolesc Med 1998;152:1232-5.

29. Moser DK, Coleman S. Recommendations for improving cardiopulmonary resuscitation skills retention. Heart Lung 1992;21:372-80.

30. Berden HJ, Willems FF, Hendreick JM, Pijls NH, Knape JT. How frequently should basic cardiopulmonary resuscitation training be repeated to maintain adequate skills? BMJ 1993;306:1576-77.

31. Cummins RO, Austin D, Graves JR, Litwin PE, Pierce J. Ventilation skills of emergency medical technicians: a teaching challenge for emergency medicine. Ann Emerg Med 1986;15:1187-92.

32. Dawson JA, Schmölzer GM, Kamlin CO, Te Pas AB, O'Donnell CP, Donath $\mathrm{SM}$ et al. Oxygenation with t-piece versus self-inflating bag for ventilation of extremely preterm infants at birth: a randomized controlled trial. J Pediatr 2011;158:912-18.

33. Dawson JA, Gerber A, Kamlin CO, Davis PG, Morley CJ. Providing PEEP during neonatal resuscitation: Which device is best? J Paediatr Child Health 2011:47:698-703.

34. Deakin CD, Murphy D, Couzins M, Mason S. Does an advanced life support course give non-anaesthetists adequate skills to manage an airway? Resuscitation 2010;81:539-43

35. Klingenberg C, Dawson JA, Gerber A, Kamlin CO, Davis PG, Morley CJ. Sustained Inflations: Comparing Three Neonatal Resuscitation Devices. Neonatology 2011;26;100:78-84.

36. Nehme Z, Boyle MJ. Smaller self-inflating bags produce greater guideline consistent ventilation in simulated cardiopulmonary resuscitation. BMC Emerg Med 2009;20:9-4.

37. Resende JG, Menezes CG, Paula AM, Ferreira AC, Zaconeta CA, Silva $\mathrm{CA}$ et al. Evaluation of peak inspiratory pressure and respiratory rate during ventilation of an infant lung model with a self-inflating bag. J Pediatr 2006;82:359-64.

38. Roehr CC, Kelm M, Fischer HS, Buhrer C, Schmalisch G, Proquitte H. Manual ventilation devices in neonatal resuscitation: tidal volume and positive pressureprovision. Resuscitation 2010;81:202-5.

39. Schoenfeld PS, Baker MD. Management of cardiopulmonary and trauma resuscitation in the pediatric emergency department. Pediatrics 1993;91:726-9.

40. O'Donnell CP, Davis PG, Morley CJ. Positive pressure ventilation at neonatal resuscitation: review of equipment and international survey of practice. Acta Paediatr 2004;93:583-8.

41. O'Donnell CP, Davis PG, Lau R, Dargaville PA, Doyle LW, Morley CJ. Neonatal resuscitation 2: an evaluation of manual ventilation devices and face masks. Arch Dis Child Fetal Neonatal Ed 2005;90:392-6.

42. Barnes TA, McGarrv WP. Evaluation of ten disposable manual resuscitators. Respir Care 1990;35:960-8.

43. Barnes TA, Stockwell DL. Evaluation of ten manual resuscitators across an operational temperature range of $-18^{\circ} \mathrm{C}$ to $50^{\circ} \mathrm{C}$. Respir Care $1991 ; 36: 161-72$.

44. Mazzolini DG Jr, Marshall NA. Evaluation of 16 adult disposable manual resuscitators. Respir Care 2004;49:1509-14.

45. Field D, Milner AD, Hopkin IE. Efficiency of manual resuscitators at birth. Arch Dis Child 1986;61:300-2.

46. Finer NN, Barrington KJ, Al-Fadley F, Peters KL. Limitations of self-inflating resuscitators. Pediatrics 1986;77:417-20. 
47. Connors R, Kissoon N, Tiffin N, Frewen TC. An evaluation of the physical and functional characteristics of infant resuscitators. Ped Emerg Care 1993;9:104-7.

48. Stemp LI. Manual resuscitators and spontaneous ventilation-an evaluation. Crit Care Med 1992;20:1496.

49. Cooper RM, Grgas S. Fatal barotrauma resulting from misuse of a resuscitation bag. Anesthesiology 2000;93:892-3.

50. Hermansen MC, Prior MM. Oxygen concentrations from self-inflating resuscitation bags. Am J Perinatol 1993;10:79-80.

51. Nam SH, Kim KJ, Nam YT, Shim JK. The changes in delivered oxygen fractions using laerdal resuscitator bag with different types of reservoir. Yonsei Med $\mathrm{J}$ 2001;42:242-6.

52. Godoy AC, Vieira RJ. Comparison of the FiO2 delivered by seven models of the self-inflating bag-mask system. Rev Bras Anestesiol. 2009;59:21-7.

53. Kattwinkel J, ed. Textbook of neonatal resuscitation. $5^{\text {th }}$ ed. Elk Grove Village, IL: American Academy of Pediatrics; 2006.

54. Johnston KL, Aziz K. The self-inflating resuscitation bag delivers high oxygen concentrations when used without a reservoir: implications for neonatal resuscitation. Respir Care 2009;54:665-70.

55. Thió M, Bhatia R, Dawson JA, Davis PG. Oxygen delivery using neonatal selfinflating resuscitation bags without a reservoir. Arch Dis Child Fetal Neonatal 2010; 95:F315-9.

56. Morley CJ, Dawson JA, Stewart MJ, Hussain F, Davis PG. The effect of a PEEP valve on a Laerdal neonatal self-inflating resuscitation bag. J Paediatr Child Health 2010;46:51-6. 